\title{
Erratum
}

Behrouz Fathi-Vajargah* and Mohadeseh Kanafchian

\section{Improved Markov Chain Monte Carlo method for cryptanalysis substitution-transposition cipher}

DOI: $10.1515 / \mathrm{mcma}-2017-0108$

Erratum to: B. Fathi-Vajargah and M. Kanafchian, Improved Markov Chain Monte Carlo method for cryptanalysis substitution-transposition cipher, Monte Carlo Methods Appl. 22 (2016), no. 4, 323-335

(https://doi.org/10.1515/mcma-2016-0116).

The affiliation for Mohadeseh Kanafchian was listed incorrectly as “Department of Statistics”. For the reader's convenience, the corrected author line appears below.

Mohadeseh Kanafchian: Department of Mathematics, University of Guilan, Rasht, Iran,

e-mail: m.kanafchiyan@gmail.com

*Corresponding author: Behrouz Fathi-Vajargah: Department of Statistics, University of Guilan, Rasht, Iran, e-mail: fathi@guilan.ac.ir

Mohadeseh Kanafchian: Department of Mathematics, University of Guilan, Rasht, Iran, e-mail: m.kanafchiyan@gmail.com 